Encontros Bibli: revista eletrônica de biblioteconomia e ciência da informação, $v .17, n$. esp. 2 - III SBCC, p.19-35, 2012. ISSN 1518-2924. DOI:10.5007/15182924.2012v17nesp $2 p 19$

\title{
MAPEAMENTO DA PRODUÇÃO CIENTÍFICA BRASILEIRA SOBRE ACESSO ABERTO: 2001 A 2011
}

\author{
Fábio Mascarenhas e Silva \\ Natanael Vitor Sobral ${ }^{\text {i }}$ \\ Guilherme Alves Santana ${ }^{\text {ii }}$ \\ Tatyane Lucia Cruz ${ }^{\text {iv }}$
}

\begin{abstract}
Resumo: A avaliação da produção científica permite um entendimento sobre a análise de temáticas, o mapeamento de comportamentos e tendências da comunidade de pesquisa, a identificação de oportunidades para o desenvolvimento de áreas, assim como a investigação de campos do conhecimento já consolidados ou estagnados. Neste ínterim, o presente trabalho objetiva sistematizar parte da produção científica brasileira sobre Acesso Aberto realizada entre 2001 e 2011 a partir dos dados disponíveis na Plataforma Lattes (PL) do CNPq. Para tanto, utilizaram-se softwares bibliométricos e cientométricos para a contagem e análise da produção. Dentre os principais resultados obtidos, destacam-se: identificação das tipologias documentais adotadas para a disseminação da produção de conhecimento; evolução cronológica da produção científica; e distribuição geográfica dos pesquisadores e índices de colaboração entre pesquisadores (redes sociais).
\end{abstract}

Palavras-chave: Acesso Aberto. Arquivos Aberto. Produção Científica. Plataforma Lattes (PL).

MAPPING OF BRAZILIAN SCIENTIFIC PRODUCTION ON OPEN ACCESS: 2001 TO 2011

\begin{abstract}
The evaluation of scientific understanding allows the analysis of thematic, mapping behavior and trends of the research community, identifying opportunities for the development of areas, as well as the investigation of fields of knowledge already established or stagnant. Meanwhile, this paper aims to map the Brazilian scientific production on Open Access held between 2001 and 2011, from the data available in the curriculum of Doctors of the Lattes Platform (PL). For this purpose, we used bibliometric and scientometric softwares in order to count and analyze the production. Among the main results, we highlight the types of identification documents adopted for the dissemination of knowledge production, chronological development of scientific, geographic distribution of researchers and indexes of collaboration between researchers (social networks).
\end{abstract}

Keywords: Open Access. Open Archives. Scientific Production. Lattes Database.

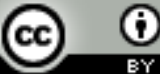

Esta obra está licenciada sob uma Licença Creative Commons

\footnotetext{
${ }^{\mathrm{i}}$ Universidade Federal de Pernambuco - fabio.mascarenhas@ufpe.br

ii Universidade Federal de Pernambuco - natan_sobral@yahoo.com.br

iii Universidade Federal de Pernambuco - guilherme.alves.santana@gmail.com

iv Universidade Federal de Pernambuco - tatyanegestaodainformacao@yahoo.com.br
} 


\section{INTRODUÇÃO}

A avaliação e o monitoramento da produção científica são atividades contributivas ao mapeamento da composição e da produtividade de pesquisadores, grupos e instituições. Igualmente, podem contribuir para a tomada de decisão e gestão de políticas públicas. Não se trata de uma tarefa trivial, uma vez que a gestão e a avaliação da atividade científica exigem a formulação de técnicas e principalmente a formação de recursos humanos habilitados para compreender os fenômenos da criação do conhecimento e como transformá-lo em resultados econômicos ou estratégicos (SANTOS, 2003).

A produção de indicadores se insere no contexto da avaliação da produção científica como um mecanismo que almeja a maximização dos esforços empreendidos pelos recursos humanos, pois pode indicar aspectos positivos e negativos desse campo de atuação. Além da avaliação da comunidade científica, os indicadores permitem identificar o grau de maturidade (evolução, estagnação ou retrocesso) de temáticas, propiciando um entendimento sobre o desenvolvimento e/ou sobre a consolidação de áreas do conhecimento.

Indicadores também podem aferir se o esforço de pesquisadores para a disseminação de uma temática está gerando resultados significativos ou não. Um bom exemplo disso são as pesquisas sobre Acesso Aberto no Brasil. Embora já haja um entendimento acerca da necessidade do acesso livre a informações científicas, não há indícios mensuráveis do impacto quantitativo desses estudos na produção científica brasileira. Nesse sentido, este trabalho objetivou sistematizar parte de produção científica brasileira sobre Acesso Aberto realizada entre 2001 e 2011 a partir dos dados coletados da Plataforma Lattes (PL) do Conselho Nacional de Desenvolvimento Científico e Tecnológico (CNPq).

\section{AVALIAÇÃO E INDICADORES DE PRODUÇÃO CIENTÍFICA}

Há uma mútua e intercambiável relação entre o setor da Ciência, da Tecnologia e da Inovação (CT\&I). Tanto em pesquisas científicas como no desenvolvimento e na inovação de produtos e processos, um dos insumos essenciais é a informação especializada, denominada Informação Científica e Tecnológica (ICT), comunicada em forma de artigos, trabalhos, patentes, relatórios, dados estatísticos, dentre outros. De acordo com o trabalho coordenado pelo Instituto Brasileiro de Informação em Ciência e Tecnologia (IBICT), a ICT representa: 
toda a informação que os cientistas e as organizações de Pesquisa e Desenvolvimento (P\&D) precisam para desenvolver suas atividades [...]; as demandadas pelas interfaces da produção científico-tecnológica com o Estado e suas instâncias decisórias, no planejamento e gestão de C\&T; e finalmente, informações destinadas a ampliar a participação da cidadania e suas expressões organizadas nos processos de elaboração de políticas públicas (GÓMEZ, CANONGIA, 2001, p.12).

O conceito de ICT proposto pelo IBICT compreende as informações que apoiam o planejamento e a gestão da CT\&I, e ainda servem como instrumento de compartilhamento de novos conhecimentos. Para avaliar esse escopo informacional há técnicas e instrumentos abarcados no conjunto de estudos da Cientometria e Bibliometria. Segundo a FAPESP (2002), os estudos cientométricos são métodos usados para a análise do sistema de CT\&I. Assim, a cientometria se ocupa com o desenvolvimento de metodologias para a construção e análise de indicadores, com base em abordagem interdisciplinar, envolvendo a Ciência da Informação, Economia, Administração e outras áreas do conhecimento.

A cientometria engloba o estudo das ciências físicas, naturais e sociais, preocupando-se com a "dinâmica da ciência, como atividade social, tendo como objetos de análise a produção, a circulação e o consumo da produção científica" (SANTOS, KOBASHI, 2009, p. 159). Almeja, igualmente, auxiliar na compreensão da estrutura, da evolução e das conexões do universo científico, de modo a estabelecer relações das ciências com os avanços tecnológicos, econômicos e sociais. Ressalta-se, ainda, que a cientometria baseia-se em indicadores construídos a partir de documentos publicados em canais especializados e envolve inúmeros parâmetros, tais como a quantidade de publicações, co-autorias, citações, co-ocorrência de palavras e outros (FAPESP, 2002).

Já a bibliometria pode ser entendida como um conjunto de técnicas e métodos quantitativos para a gestão de instituições envolvidas com o tratamento de informação (PRITCHARD, 1969). Na gestão da informação e no planejamento científico e tecnológico, sua aplicabilidade se verifica na avaliação da produtividade de pesquisadores, na identificação dos centros de pesquisa mais desenvolvidos e no reconhecimento da consolidação de uma área científica (BORSCHIVER, GUEDES, 2005).

Portanto, a Bibliometria e a Cientometria auxiliam diretamente na avaliação da ciência e contribuem consideravelmente para a análise e elaboração de indicadores de desempenho. Sobre o conceito e a função de indicadores, observa-se que estes são, em primeira definição, dados estatísticos usados para avaliar as potencialidades da base científica e tecnológica dos países. São capazes também de monitorar as oportunidades em diferentes áreas e identificar 
atividades e projetos mais promissores para o futuro, auxiliando as decisões estratégicas dos gestores da política científica e tecnológica e o conhecimento, por parte da comunidade científica, do sistema no qual está inserida.

Evidencia-se que o uso dos indicadores auxilia o processo de avaliação de qualquer área do conhecimento, no sentido de responder questionamentos acerca da difusão, dos crescimentos e declínios de determinadas temáticas. Nesse ínterim, é perceptível que os estudos e pesquisas sobre Acesso Aberto venham se expandindo ao longo da última década, mas ainda não se sabe se estão consolidados. Tal temática compreende aspectos como o acesso à informação, repositórios e bibliotecas digitais, gratuidade no acesso a bases de dados e periódicos científicos internacionais, perpassando também pela elaboração de políticas públicas, metodologias, técnicas e constituição da fundamentação teórico-conceitual.

Partindo desse pressuposto, infere-se que a elaboração de indicadores científicos sobre o Acesso Aberto no Brasil pode evidenciar qual a situação atual da produção científica brasileira sobre a temática. Portanto, identificar e analisar o comportamento da produção científica dessa temática contribui para as atividades de investigação científica da área, servindo também para o resgate da memória científica institucional, bem como para processos de tomada de decisão, criação de políticas em CT\&I e fomento para pesquisas na área.

\section{A Plataforma LATtes COMO BASE PARA A CONSTRuÇÃo DE INDICADORES CIENTÍFICOS}

No processo de análise das informações científicas para a produção de indicadores, encontrar a fonte de dados primários, em suporte digital, mais adequada às necessidades de produção dos indicadores é a parte mais difícil. Em geral, as bases de dados não foram construídas para a produção dos indicadores, havendo necessidade de reformatar os campos antes da importação dos dados, o que sempre é uma operação onerosa (SANTOS, 2003; QUONIAM, 1992).

Uma vez que o governo e instituições reguladoras da atividade científica do país ocupam posições estratégicas na criação de políticas e gestão da ICT, elas deveriam se responsabilizar pela sistematização e integração de bases de dados para a formulação de indicadores. Contudo, bases brasileiras com dados de produção científica ainda não são plenamente integradas e carecem de maior abrangência e exaustividade sobre o conhecimento 
produzido pelos pesquisadores do país. Já em 2004, Mugnaini, Jannuzzi e Quoniam defendiam ser importante a investigação da produção da CT\&I brasileira em bases bibliográficas e citaram incipientes esforços do Scielo e da Plataforma Lattes (PL) do CNPq para a geração de indicadores.

Por ser um sistema integrado de informações gerenciais, a PL se insere como uma base de dados que pode atuar como instrumento fundamental para as atividades de geração de indicadores de produção científica, possibilitando o tratamento e a difusão das informações necessárias à formulação e à gestão de políticas de Instituições de Ensino Superior (IES).

Ao longo dos anos a PL se consolidou como um importante repositório de informações relevantes à memória científica e tecnológica nacional. Isso possibilitou maior profissionalização e padronização da gestão da informação do Sistema Nacional de CT\&I à medida que a plataforma foi sendo reconhecida em todo cenário nacional como instrumento de apoio às políticas do setor. Com isso, o papel da PL foi se expandindo, e sua utilidade à tomada de decisão no âmbito da gestão de CT\&I conquistou maior maturidade, permitindo que os gestores do setor consultassem informações na base e aplicassem metodologias para a obtenção de indicadores de caráter decisório, subsidiando o planejamento e a execução de suas atividades (SANTANA et al, 2011).

Dessa maneira, gestores de instituições podem fazer uso das informações que estão contidas nos currículos Lattes de pesquisadores, de modo que seus desempenhos possam ser avaliados para a maximização de seus esforços e recursos. Em nosso caso, percebemos um grande potencial na construção de uma metodologia de geração de indicadores científicos fundamentados nos dados da base de currículos da PL, em parte pelo fato de haver constantes exigências de órgãos de pesquisa para que os pesquisadores atualizem os dados referentes à sua atuação. Cabe, no entanto, destacar que os dados da PL não são disponibilizados em estrutura apropriada para a produção de indicadores, logo, torna-se necessário empreender esforços para coleta e re-organização dos dados, adotando ferramentas e técnicas bibliométricas e cientométricas ad hoc, específicas para reformatar e padronizar dados estatísticos. 


\section{PROCEDIMENTOS METODOLÓGICOS}

Para identificar publicações sobre a temática Acesso Aberto no Brasil fez-se uma busca na Plataforma Lattes (PL). No processo de busca, em razão da sinonímia, além da expressão Acesso Aberto, foram incluídas: Acesso Livre (também em inglês: Open Access, Open Archives) e Arquivos Abertos (também em espanhol: Acesso Abierto e Archivo Abierto) no campo Assunto. Optou-se por analisar os currículos dos pesquisadores com título de doutor que publicaram entre os anos de 2001 a 2011. O resultado inicial recuperou 197 pesquisadores que haviam registrado em seus respectivos currículos (nos campos de palavraschave, projetos de pesquisa, áreas de atuação ou nas referências dos artigos ou trabalhos) ao menos um dos termos da estratégia de busca.

Dos 197 pesquisadores do resultado inicial, apenas 82 publicaram sobre a temática do corpus desta pesquisa, ou seja, sobre o acesso aberto no contexto da comunicação científica, excluindo-se, por exemplo, trabalhos que relatavam o acesso aberto de animais ao pasto. Para coletar os currículos foi adotada a ferramenta ScriptLattes, especializada em extração de dados da base de currículos do CNPq, que compilou e efetuou o cruzamento de referências entre as publicações (eliminando as produções duplicadas) dos pesquisadores. Ainda coletaram-se outros dados, como: área do conhecimento do Doutorado; vínculo institucional; e se era Bolsista de Produtividade em Pesquisa.

Em seguida, estratificaram-se os anos de publicação segundo as tipologias documentais: Artigos completos publicados em periódicos; Livros publicados/organizados; Capítulos de livros publicados; Trabalhos completos; Resumos expandidos; e Resumos publicados em anais de eventos. Utilizou-se o Microsoft Excel para a tabulação dos dados das produções e posterior geração dos gráficos de produção científica.

Os dados de autoria foram inseridos em arquivos de texto e foram submetidos à configuração, extração e inversão no software DataView, que gerou matrizes quadradas de colaboração entre os autores e fez a contagem do número de publicações realizadas por cada pesquisador. A partir das matrizes geradas no DataView, elaborou-se um gráfico de relações entre os autores no software UCInet. Por meio dessas matrizes, criaram-se atributos numéricos representativos da frequência de textos publicados por cada autor. As matrizes geradas foram processadas pelo software NetDraw (incluso no UCInet), permitindo a criação de gráficos capazes de relacionar a colaboração entre instituições e autores doutores. 
Em síntese, os procedimentos e softwares utilizados permitiram gerar indicadores sobre: produção científica média e total; evolução cronológica da produção científica; distribuição geográfica dos pesquisadores; instituições com maior número de pesquisadores da temática; índices de colaboração entre pesquisadores (redes sociais). Esses indicadores serão apresentados na próxima seção deste trabalho.

\section{ANÁLISE E DISCUSSÃO DOS RESULTADOS}

As análises a seguir centraram-se nas áreas de formação de origem dos autores, nas regiões geográficas e respectivas instituições às quais os pesquisadores estavam vinculados no período de elaboração deste trabalho (abril de 2012), na produção por tipologia distribuída por anos, e numa rede social elaborada a partir das relações de produção entre os pesquisadores.

O Gráfico 1 representa as áreas de conhecimentos nas quais os autores titularam-se como doutores.

Gráfico 1 - Área de Conhecimento do Doutorado dos pesquisadores.

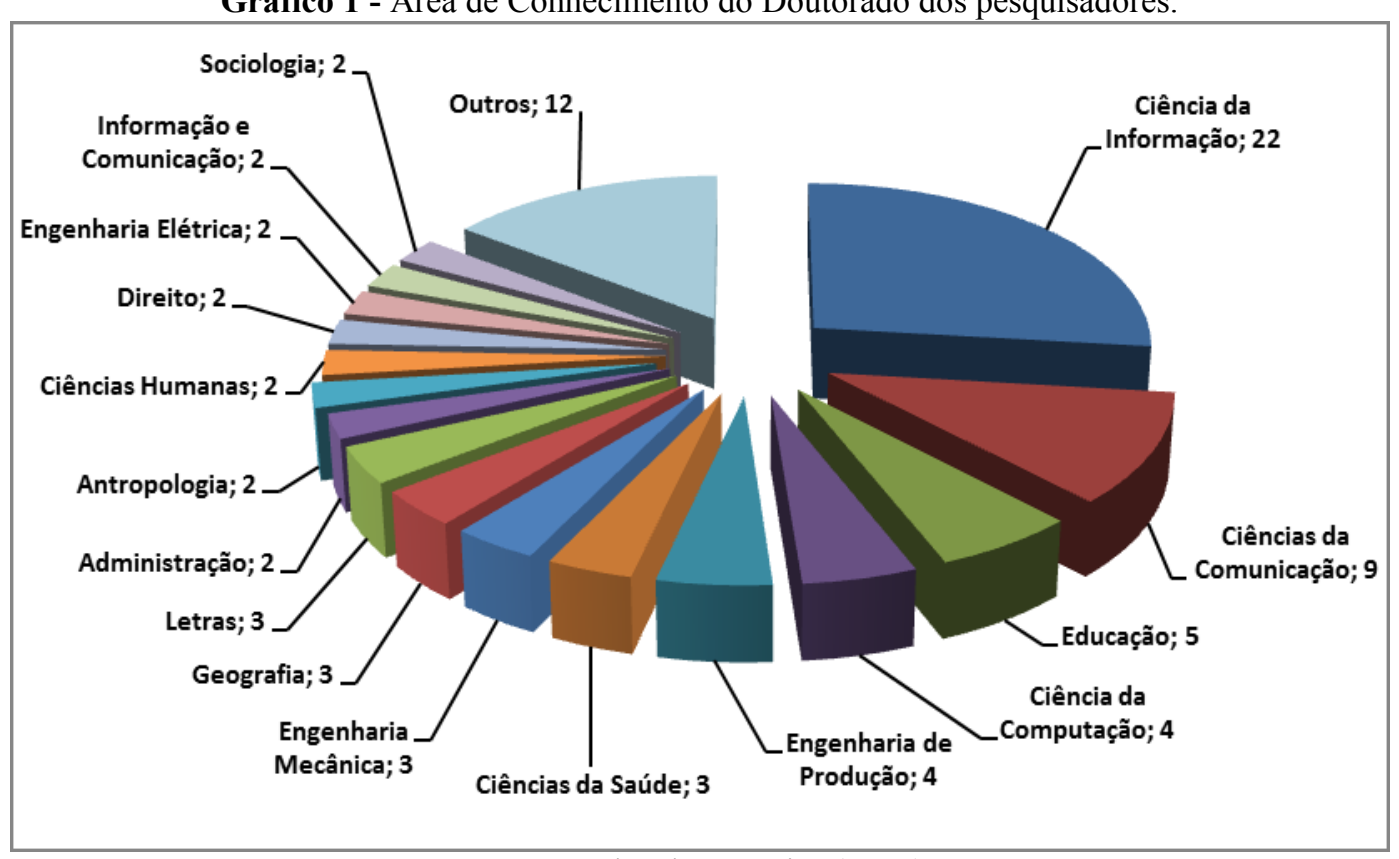

Fonte: Dados da Pesquisa (2012).

Nota-se que a maioria dos Autores $(26,83 \%)$ concluiu o doutorado na área de Ciência da Informação; tal concentração reforça o interesse da área por abordagens que tratem do uso social da informação, da comunicação científica e da produção da Informação. Ainda vale 
destacar a contribuição da área das Ciências da Comunicação para a propagação da temática, enfaticamente nas questões relativas à divulgação da informação e do conhecimento. Ainda, às áreas de Educação e Ciência da Computação cabem discussões pertinentes ao acesso, disponibilização e recuperação da informação, ao uso da informação para o desenvolvimento social e também ao acesso ao conhecimento para a promoção do saber. Ressalta-se que a categoria OUTROS representa as áreas com apenas uma incidência.

Além da formação, identificaram-se as instituições às quais os pesquisadores estavam vinculados e as regiões e estados brasileiros onde havia maior concentração de autores. A Figura 1 expõe a distribuição geográfica dos vínculos institucionais dos autores e os polos de concentração dos pesquisadores que publicaram textos sobre Acesso Aberto entre 2001 e 2011. Ressalva-se que, por estar vinculado a uma instituição estrangeira, um autor não foi incluído.

Figura 1 - Distribuição Geográfica dos Autores sobre Acesso Aberto.

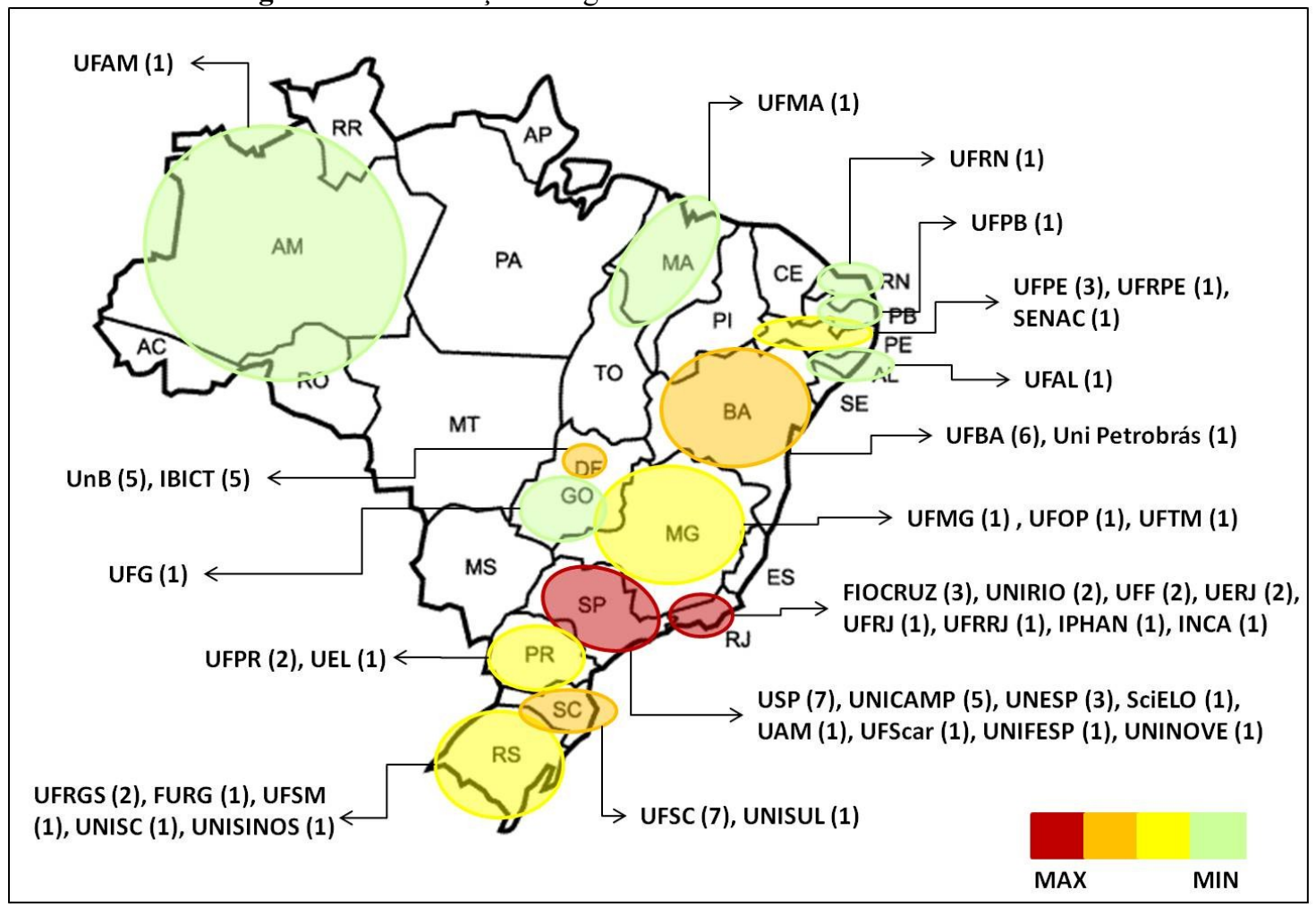

Fonte: Dados da Pesquisa (2012).

A Figura 1 demonstra o considerável peso das instituições públicas de ensino na produção científica em nosso país, corroborando com a constatação de boa parte da literatura que trata de aspectos sociológicos da ciência, a qual caracteriza a pesquisa brasileira como sendo universitária e financiada pelo governo. Sob a ótica de distribuição geográfica, a concentração de autores sobre Acesso Aberto está na região Sudeste, que contabilizou 36 
pesquisadores, da qual se destacou o estado do Rio de Janeiro, com a FIOCRUZ (3 pesquisadores), UNIRIO, UFF e a UERJ (estas últimas com 2 pesquisadores); e o estado de São Paulo, com foco para a USP (7 pesquisadores), UNICAMP e UNESP (com respectivamente 5 e 3 doutores). Os estados de Santa Catarina (UFSC, com 7 doutores), Distrito Federal (IBICT e UnB, cada um com 5 pesquisadores) e Bahia (UFBA, com 6) surgem em segunda posição na produção de textos sobre o tema.

Ainda sobre a distribuição dos pesquisadores por vínculo institucional, o Gráfico 2 apresenta, em ordem crescente, quais são as instituições com o maior número de autores sobre a temática do Acesso Aberto.

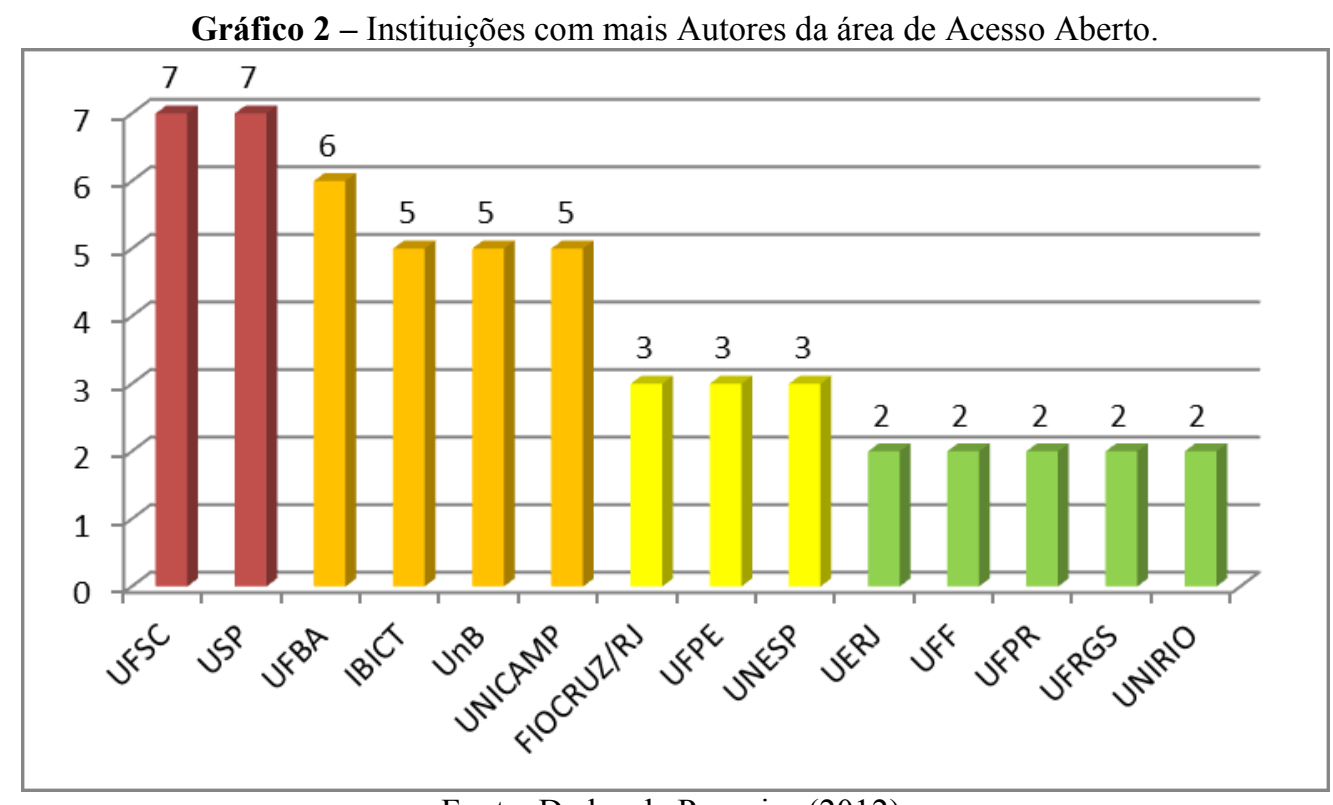

Fonte: Dados da Pesquisa (2012).

Após breve análise das áreas do conhecimento, distribuição geográfica e instituições de vínculo dos autores que escrevem sobre Acesso Aberto, enfatizar-se-ão os aspectos relacionados à produção bibliográfica destes no período de análise do corpus (2001 a 2011).

Percebeu-se que os autores publicaram 121 documentos relativos a temática Acesso Aberto entre 2001 e 2011 (ver Quadro 2). Dentre as tipologias mais representativas deste total, destacam-se as produções de trabalhos completos publicados em anais de eventos (representando quase metade da produção total) e de artigos publicados em periódicos $(26,45 \%$ do total geral). 
Quadro 2 - Produção Geral Total por Tipologia Documental.

\begin{tabular}{|l|c|c|}
\hline \multirow{2}{*}{\multicolumn{1}{|c|}{ Tipologia }} & \multicolumn{2}{c|}{ Total } \\
\cline { 2 - 3 } & $\sum$ & \% \\
\hline Trabalhos Completos & 56 & $46,28 \%$ \\
\hline Artigos Completos & 32 & $26,45 \%$ \\
\hline Capítulos de Livros & 14 & $11,57 \%$ \\
\hline Resumos & 10 & $8,26 \%$ \\
\hline Resumos Expandidos & 7 & $5,79 \%$ \\
\hline Livros Publicados & 2 & $1,65 \%$ \\
\hline Total Geral & $\mathbf{1 2 1}$ & $\mathbf{1 0 0 \%}$ \\
\hline
\end{tabular}

Fonte: Dados da Pesquisa (2012).

Verificou-se também que 14 produções se destinaram a Capítulos de Livros e 10 a resumos de anais de eventos. Na produção em anais constam eventos do Brasil e do Exterior, com ênfase na Conferência Luso-Brasileira sobre Acesso Aberto (CONFOA) e no Simpósio Brasileiro de Comunicação Científica (SBCC).

A seguir, os Gráficos 3 e 4 apresentam respectivamente a produção total em cada tipologia e a evolução das publicações durante o período pesquisado.

Gráfico 3 - Produção Total por Tipologia Documental

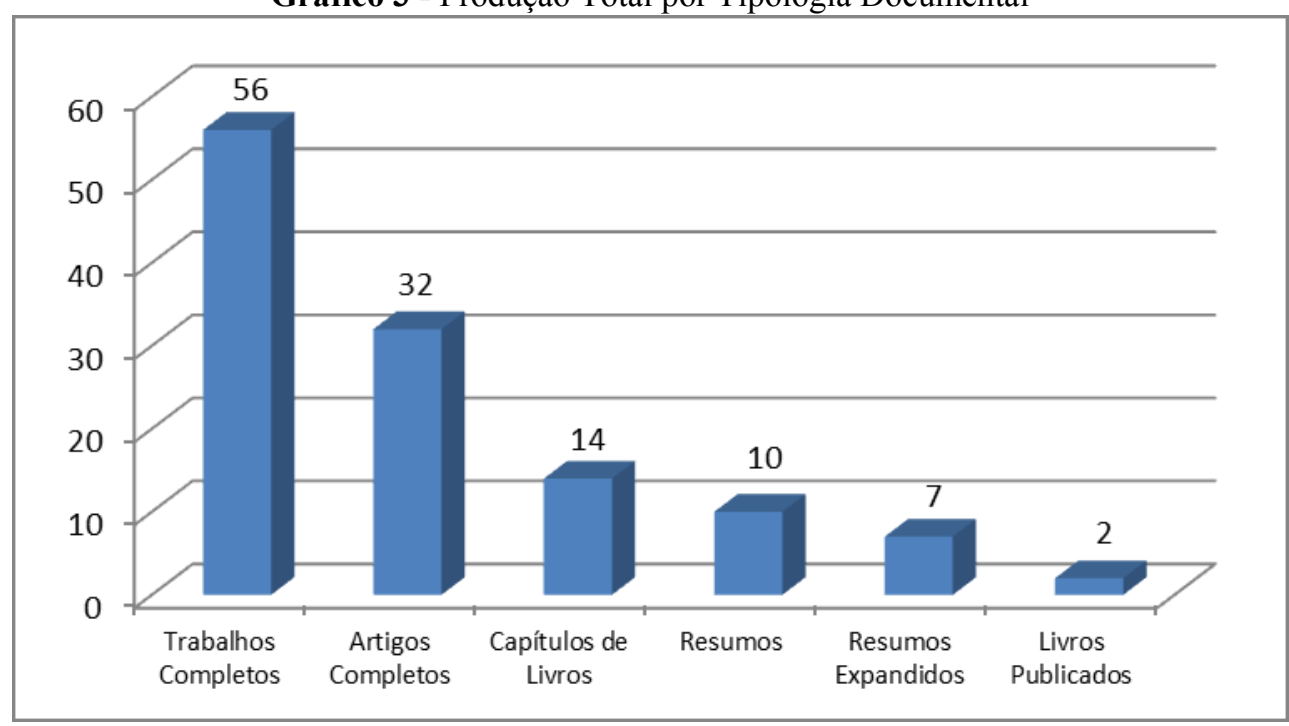

Fonte: Dados da Pesquisa (2012). 


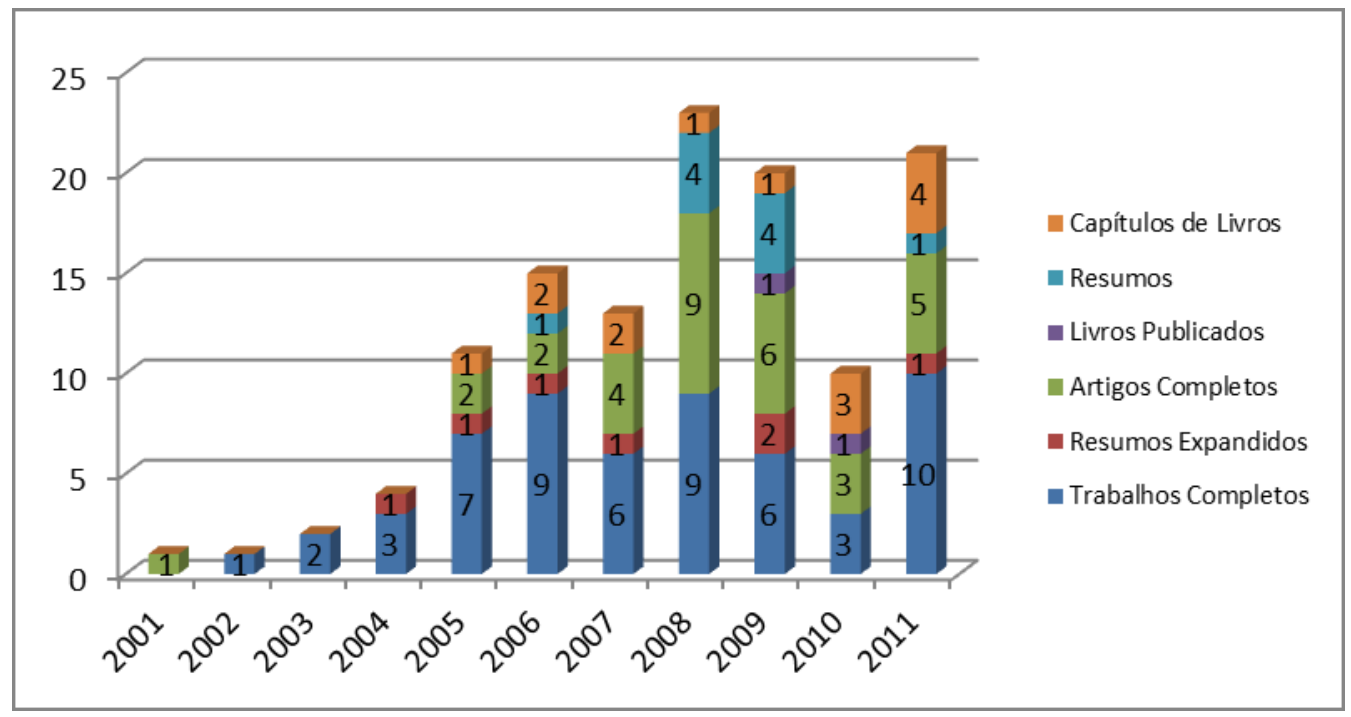

Fonte: Dados da Pesquisa (2012).

Observa-se no Gráfico 4 o crescimento da produção sobre Acesso Aberto entre 2001 e 2011. Esse crescimento acentua-se a partir de 2005, onde se constatou uma maior diversidade de tipologias documentais sobre a temática. Chama a atenção o ano de 2008 como o ano mais produtivo (23 produções), possivelmente em razão do despertar do interesse nas pesquisas sobre o assunto e/ou da consolidação de grupos de pesquisa e oferta de eventos técnicocientíficos. Por outro lado, há acentuada queda nas produções em 2010, mas para inferir as razões dessa redução, sugere-se estudo mais minucioso. Entretanto, a queda registrada nas produções em 2010 foi superada em 2011 com o número de publicações superior ao dobro das publicações do ano anterior (de 10 para 21$)$.

Em seguida tratar-se-á das redes sociais constituídas a partir do corpus de atores delimitados neste estudo. Para tal fez-se o uso de sociogramas, que são representações da aplicação da técnica de Análise de Redes Sociais (ARS) para o estudo do Sistema de CT\&I. Estes possuem caráter de indicadores, pois apontam comportamentos e padrões não detectáveis em outras técnicas. Entretanto, a combinação da ARS com outras metodologias proporciona resultados mais satisfatórios.

Silva et al. (2006) destaca que a ARS permite uma análise mais aprofundada da colaboração entre os docentes, ressaltando aspectos que não seriam detectáveis com o uso de métodos estatísticos tradicionais. Dessa forma, defende-se que a ARS engloba um conjunto de procedimentos e aplicações com potencial de contribuição para estudos métricos da informação, especialmente aqueles direcionados à análise da produção científica e de redes de colaboração científica. 
O Gráfico 5 remete à análise de redes de colaboração dos pesquisadores do corpus. Cada ator que compõe a rede foi representado com círculo ou losango (que indicam que o pesquisador possui bolsa de produtividade do $\mathrm{CNPq}$ vigente). As cores das formas geométricas distinguem as instituições às quais estão vinculados. A espessura das ligações, ou seja, das linhas que ligam um ator a outro determina a intensidade da relação entre eles (no que tange as publicações); quanto mais larga a ligação, mais trabalhos em colaboração foram publicados sobre a temática de Acesso Aberto. A ausência de ligações em torno de qualquer ator indica que toda a sua produção identificada no corpus desta análise foi realizada de forma individual ou com pesquisadores que não foram contemplados no corpus. Sobre esse dado, percebe-se que 26 atores aparecem isolados.

Gráfico 5 - Redes de Colaboração dos Pesquisadores de Acesso Aberto.

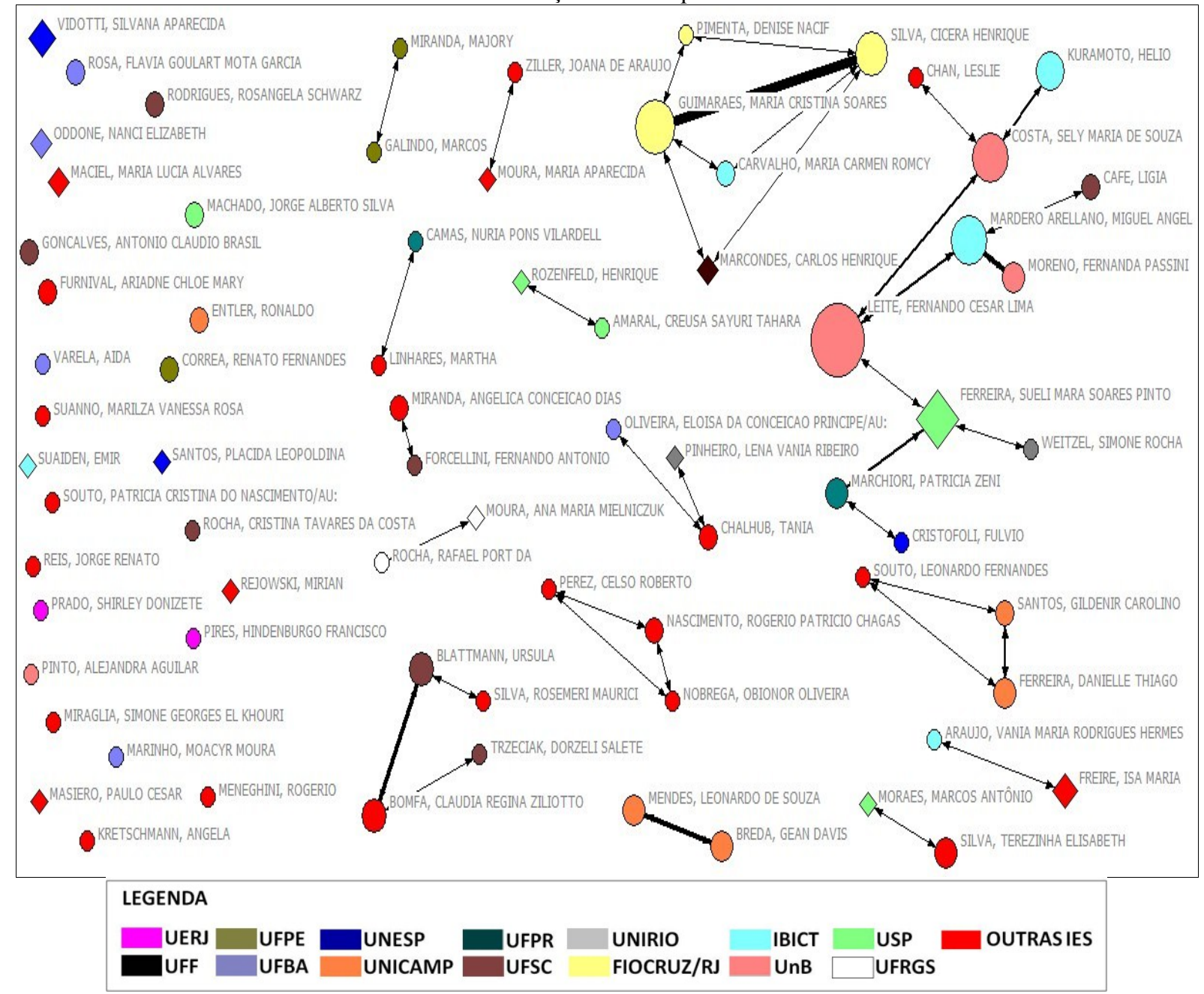

Fonte: Dados da Pesquisa (2012).

Para a análise do Gráfico 5 adotou-se o conceito de relações intraorganizacionais e interorganizacionais, tomando-se por parâmetro a instituição dos autores. Com isso as 30

Enc. Bibli: R. Eletr. Bib. Ci. Inf., ISSN 1518-2924, Florianópolis, v. 17, n. esp. 2 - III SBCC, p.19-35, 2012 
relações entre membros da mesma instituição foram classificadas como intraorganizacionais, e as configuradas entre membros de instituições diferentes foram denominadas interorganizacionais. Notou-se que há 15 grupos de autores que publicaram entre si, estabelecendo redes sociais. Desses grupos, 6 são intraorganizacionais e 9 são interorganizacionais, o que indica que $40 \%$ das sub-redes existentes são fechadas entre integrantes da mesma instituição, e as outras $60 \%$ contam com pesquisadores de mais de uma instituição.

Para uma compreensão da dinâmica da produção científica sobre Acesso Aberto no Brasil, a representação das colaborações entre docentes na formulação dos trabalhos não poderia deixar de ser contemplada. Para que as análises fossem realizadas de forma mais clara, a amostra inicial de 82 pesquisadores foi delimitada a 28 . O critério adotado foi a quantidade de colaborações, segundo o qual foram incluídas apenas as sub-redes com mais de 3 pesquisadores.

Gráfico 6 - Medidas de Centralidade nas Redes de Colaboração dos Pesquisadores de Acesso Aberto.

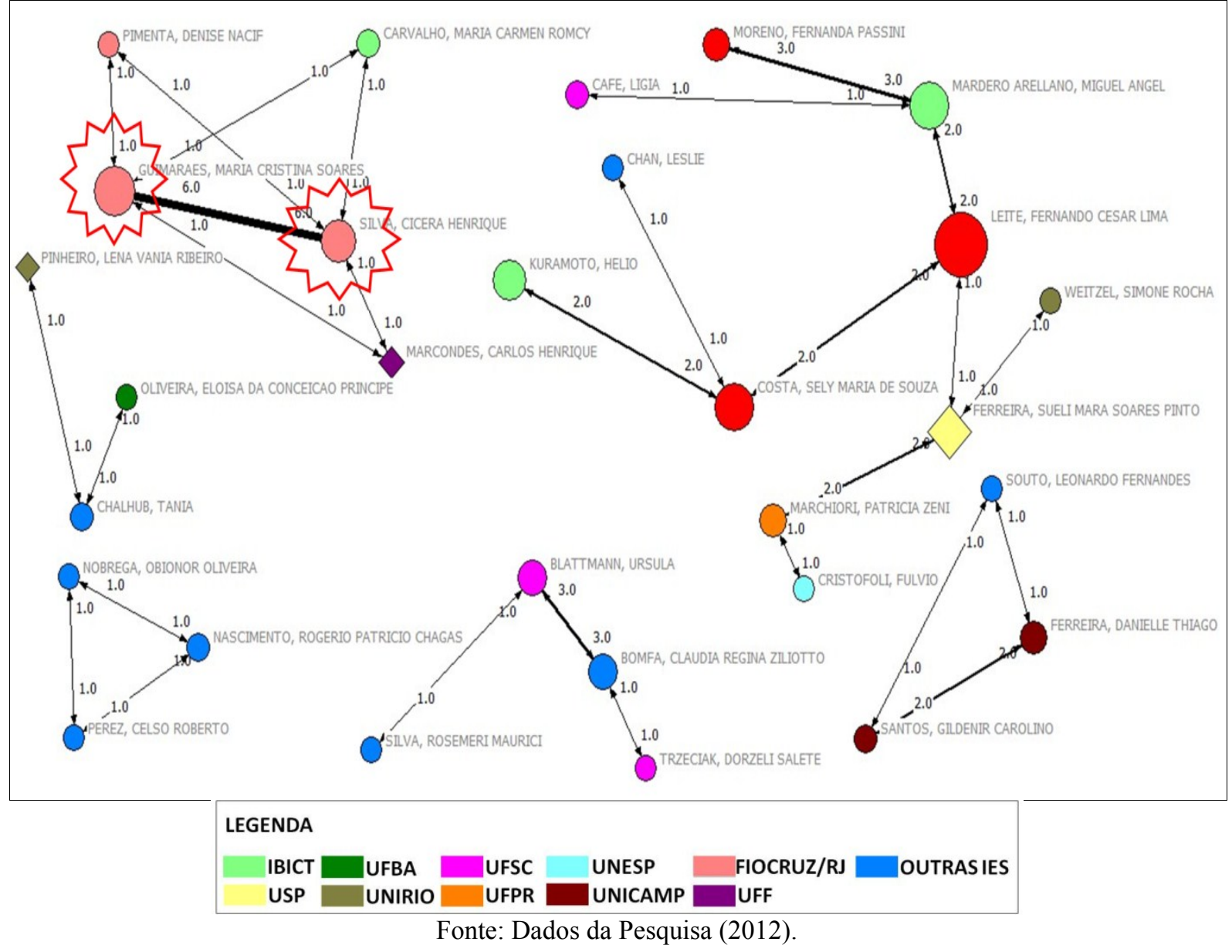


A partir do Gráfico 6 é possível visualizar melhor atores centrais da rede, ou seja, aqueles que se destacaram por agregar pesquisadores com o intuito de produzir conhecimentos sobre Acesso Aberto, e ainda por manter mais vínculos com outros atores. Foram eles: SILVA, Cícera Henrique; e, GUIMARÃES, Maria Cristina Soares (ambas em destaque no grafo com a estrela vermelha) figurando com 4 relações cada, sendo assim as que possuem mais vínculos; depois, aparecem COSTA, Sely Maria de Souza; LEITE, Fernando Cesar Lima; MARDERO, Arellano Miguel Angel; e FERREIRA, Sueli Mara Soares Pinto, com 3 relações cada.

Outros importantes aspectos analisados foram o quantitativo da produção de cada docente e a intensidade de suas relações nas publicações científicas. No que diz respeito ao quantitativo, quanto mais publicações sobre Acesso Aberto o pesquisador apresentava, maior era sua figura geométrica no grafo de colaboração (Gráfico 6). Assim, LEITE, Fernando Cesar Lima é o pesquisador mais produtivo, com 12 publicações específicas sobre o tema, seguido de GUIMARAES, Maria Cristina Soares; e FERREIRA, Sueli Mara Soares Pinto, que figuram com 8 publicações cada; MARDERO ARELLANO, Miguel Angel; e COSTA, Sely Maria De Souza, que aparecem com 7; SILVA, Cicera Henrique com 6; e KURAMOTO, Helio com 5.

Quanto ao aspecto intensidade da relação, detectou-se que a parceria que gerou mais publicações foi a da GUIMARAES, Maria Cristina Soares \& SILVA, Cicera Henrique (6 produções em parceria). Ainda constam as relações: MORENO, Fernanda Passini \& MARDERO ARELLANO, Miguel Angel; BOMFA, Claudia Regina Ziliotto \& BLATTMANN, Úrsula; e, BREDA, Gean Davis \& MENDES, Leonardo de Souza (Cada dupla com 3 produções em parceria).

\section{CONSIDERAÇÕES FINAIS}

O presente trabalho utilizou-se dos métodos bibliométricos e cientométricos e da técnica de ARS com a finalidade de prover um panorama a partir dos indicadores científicos da produção sobre Acesso Aberto no Brasil. Sem a existência de uma base de dados de abrangência nacional em C\&T, como a PL, seria difícil coletar e processar os dados, e extrair fragmentos de evidências que se revelam na forma de indicadores.

As análises apresentaram um breve recorte dos estudos sobre Acesso Aberto, demonstrando ser esse um tema de maior interesse para pesquisadores atuantes na área da 32

Enc. Bibli: R. Eletr. Bib. Ci. Inf., ISSN 1518-2924, Florianópolis, v. 17, n. esp. 2 - III SBCC, p.19-35, 2012. 
Ciência da Informação, o que não surpreende em razão da ampla participação de profissionais da informação que tem defendido e debatido a causa.

Com relação à geografia da produção sobre o assunto, destacou-se a região Sudeste, favorecida pelo bom desempenho da USP e da UNICAMP, comprovando a qualidade acadêmica dessas instituições. Esse comportamento já havia sido identificado em estudos anteriores, quando a temática em questão foi a Preservação Digital (consultar o trabalho de Silva et al. 2011b). Como o presente estudo contemplou exclusivamente doutores, outro dado que auxilia no entendimento desta desigualdade pode ser encontrado no Painel Lattes do $\mathrm{CNPq}^{3}$, onde se verifica que a região Sudeste do país possui mais doutores do que a soma de todas as outras regiões juntas, fato este que contribui para a explicação de tal destaque.

No aspecto "tipologia documental" notou-se uma baixa produção de resumos, muito provavelmente pelo fato de parte dos pesquisadores avaliados estarem vinculados a Programas de Pós-Graduação avaliados pela Coordenação de Aperfeiçoamento de Pessoal de Nível Superior (CAPES). Sabe-se que os resumos não tem peso significativo no processo avaliativo. Outra consideração importante é que a quantidade de redes sociais interorganizacionais é maior do que as redes intraorganizacionais, demonstrando a existência de colaboração entre instituições diferentes, fato que indica que a colaboração é um aspecto favorável para um futuro crescimento da produção nacional sobre o Acesso Aberto.

Sobre as limitações e dificuldades deste estudo, destaca-se a morosidade na coleta e organização dos dados decorrentes da ausência de sistemas automatizados que lidem com a produção científica nacional, problema esse que dificulta a geração de indicadores científicos em nosso país. Outra limitação do estudo diz respeito ao recorte de atores analisados e à ausência de discussões mais aprofundadas. Contudo, a proposta preliminar foi traçar um panorama global das produções no intuito de servir a futuros trabalhos.

Como proposta futura, pretende-se realizar estudos sobre outras temáticas relevantes à área de Ciência da Informação, buscando entender como se institucionalizaram e evoluíram por meio de seus aspectos produtivos. Em próximos estudos, outros indicadores podem ser empregados visando medir a qualidade da produção. Para isso, a utilização da base Web Qualis ${ }^{4}$ surge como uma alternativa de averiguar não só aspectos quantitativos e sociais da produção, mas também seus aspectos qualitativos.

\footnotetext{
${ }^{4}$ http://qualis.capes.gov.br/webqualis/
} 


\section{REFERÊNCIAS}

BORSCHIVER, S.; GUEDES, V. L. S. Bibliometria: uma ferramenta estatística para a gestão da informação e do conhecimento, em sistemas de informação, de comunicação e de avaliação científica e tecnológica. In: VI Encontro Nacional da Ciência da Informação, 2005, Salvador. Anais do CINFORM VI, 2005.

FUNDAÇÃO DE AMPARO À PESQUISA DO ESTADO DE SÃO PAULO (FAPESP). Indicadores de ciência, tecnologia e inovação em São Paulo: 2001. Organização de Francisco Romeu Landi. São Paulo: FAPESP, 2002. 488p.

GOMEZ, M. N. G. de; CANONGIA, C. (Org.). Contribuição para políticas de Informação Científica e Tecnológica. Brasília: IBICT, 2001.

MUGNAINI, R., JANNUZZI, P., QUONIAM, L. Indicadores bibliométricos da produção científica brasileira: uma análise a partir da base Pascal. Ciência da Informação, Brasília, v. 33, dez. 2004.

PRITCHARD, A. Statistical bibliography or bibliometrics. Journal of Documentation, [S. 1.], v. 25, n. 4, p.348-349, 1969.

QUONIAM, L. Bibliométrie sur des références bibliographiques: méthodologie. In : DESVALS, H.; DOU, H. (Org.) La veille technologique: l'information scientifique, technique et industrielle. Paris: Dunod, 1992. p. 243-262.

SANTANA, G. A. et al. Indicadores Científicos: Uma Análise da Produção do Programa de Pós-Graduação em Sociologia (PPGS) da UFPE a partir dos currículos da Plataforma Lattes (PL). In: XIV Encontro Regional de Estudantes de Biblioteconomia, Documentação, Ciência da Informação e Gestão da informação, 2011, São Luís-MA. Anais do XIV EREBD. São Luís-MA, 2011.

SANTOS, R. N. M.. Produção Científica: Por que medir? O que medir?. Revista Digital de Biblioteconomia e Ciência da Informação, Campinas, v. 1, n. 1, p. 22-38, jul./dez. 2003.

SANTOS, R. N. M.; KOBASHI, N. Y. Bibliometria, cientometria, informetria: conceitos e aplicações. Ciência da Informação, Brasília, v. 2, n. 1, p.155-172, jan./dez. 2009.

SILVA, A. B. O. et al. Análise de redes sociais como metodologia de apoio para a discussão da interdisciplinaridade na ciência da informação. Ciência da Informação, Brasília, v. 35, n. 1, p. 72-93. jan./abr. 2006.

SILVA, F. M.; FERREIRA, M. H. W. Indicadores Tecnológicos: uma análise da produção tecnológica dos Programas de Pós-Graduação das Engenharias da UFPE. In: SILVA, F. M. et al. A gestão da informação na era do conhecimento. Recife: NECTAR, 2011. p. 1-16.

SILVA, F. M. et al. Estratégias metodológicas para a geração de indicadores científicos: uso da Plataforma Lattes. In: ENCONTRO NACIONAL DE PESQUISA EM CIÊNCIA DA INFORMAÇÃO, 12, 2011, Brasília. Anais... Brasília, 2011a. 
SILVA, F. M. et al. Preservação digital: mapeamento da produção científica brasileira de uma década (2001-2010). In: Conferência sobre Tecnologia, Cultura e Memória, 2011, Recife. Anais do CTCM, 2011b.

SILVA, F. M.; SANTANA, G. A. Produção científica da UFPE na década de 2001 a 2010: análise e elaboração de catálogo de indicadores. Relatório de Pesquisa. 2011.

SILVA, F. M.; SOBRAL, N. V. Análise de Redes Sociais: um estudo sobre os Programas de Pós-Graduação em Administração do estado de Pernambuco. In: SILVA, F. M. et al. A gestão da informação na era do conhecimento. Recife: NECTAR, 2011. p. 1-16. 